\title{
THE TERMINAL BEHAVIOUR OF A UNIFORMLY SHORTENING PENDULUM
}

\author{
M. N. BREARLEY \\ (Received 7 July 1967)
}

\section{Introduction}

In this paper, necessary and sufficient conditions are established for the continuation of small oscillations of a simple pendulum as its string length is decreased uniformly to zero, and the accompanying terminal value of the angular displacement is determined.

In an earlier paper [1] an analysis was made of the small oscillations of an undamped simple pendulum, of initial length $l$, for which the free string length at time $t$ is

$$
r=V t+l \text {, }
$$

where $V$ is a constant, with $V \gtrless 0$ corresponding respectively to a lengthening and shortening string. The angular displacement of the pendulum was found to be

$$
\theta=x^{-1}\left[c_{1} J_{1}(x)+c_{2} Y_{1}(x)\right],
$$

where

$$
x= \pm(2 / V)(g r)^{\frac{1}{2}},
$$

the negative sign referring to a shortening string.

An initial condition necessary for the continuation of small oscillations as $r \rightarrow 0$ is

$$
t=0, \theta=0, x=x_{1}=-(2 / V)(g l)^{\frac{1}{2}},
$$

where $x_{1}$ is a zero of $J_{1}(x)$, since this precludes from (2) the term which is unbounded as $r \rightarrow 0$ and reduces it to

$$
\theta=c_{1} x^{-1} J_{1}(x) .
$$

It was shown in [1] that if the angular amplitudes remain small as $r$ decreases in the range $0 \leqq r \leqq l$ they are given by

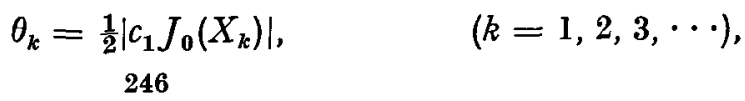


where the $X_{k}$ are successive zeros of $J_{2}(x)$ as $x$ decreases in the range $0<x<x_{1}$, and that

$$
\theta \sim \frac{1}{2} c_{1}
$$

$$
r \rightarrow 0 \text {. }
$$

It is now desired to augment (4) by a boundary condition which is sufficient to ensure the continuation of small angular displacements, (a) at each of the extrema $\theta=\theta_{k}$ of the displacement in the range $0<r \leqq l$, (b) throughout the whole of the closed range $0 \leqq r \leqq l$ of the string length.

\section{A second boundary condition for small angular amplitudes}

It is not difficult to prescribe a second initial condition appropriate for small amplitudes at extrema of $\theta$, but it is simpler to use the non-initial condition

$$
\theta=\theta_{1}, x=X_{1},
$$

where $\theta_{1}$ is an assigned angle and $X_{1}$ is the first zero of $J_{2}(x)$ encountered as $x$ decreases in the range $0<x<x_{1}$. In virtue of (6), this amounts to prescribing the amplitude of $\theta$ at its first extremum, and shows that

$$
\left|c_{1}\right|=2 \theta_{1}\left|J_{0}\left(X_{1}\right)\right|^{-1} \text {. }
$$

If there are $n$ extrema of $\theta$ in the range $0<r \leqq l$ and the angular amplitude is small at each of them, it follows from (6) that

$$
\theta_{n}=\left|J_{0}\left(X_{n}\right) / J_{0}\left(X_{1}\right)\right| \theta_{1}=O(\delta), \quad x=X_{n},
$$

where $O(\delta)$ denotes a quantity which is 'small' in the sense required for the validity of the linearised differential equation describing the pendulum motion. The condition (10) will be satisfied if

$$
\theta_{1}=\left|J_{0}\left(X_{1}\right) / J_{0}\left(X_{n}\right)\right| O(\delta),
$$

which can evidently be arranged in practice by an appropriate limitation of the initial angular velocity.

It still remains to fill the logical gap left by the assumption that under the above conditions the angular amplitudes do remain small as $r$ decreases. Reversal of the sign of $V$ transforms a shortening pendulum to a lengthening one, and if (10) is regarded as an initial condition when $V>0$ then (11) will hold at a later extremum, since for a lengthening pendulum the angular amplitudes decrease and so remain small. This 'reversibility' argument shows that (11) will imply (10) for a shortening pendulum.

It has therefore been established via (8) and (11) that (in conjunction with the necessary condition (4)) a sufficient condition for small angular 
amplitudes of a shortening pendulum which experiences $n$ extrema in the range $0<r \leqq l$ is

$$
\theta=\left|J_{0}\left(X_{1}\right) / J_{0}\left(X_{n}\right)\right| O(\delta), \quad x=X_{1},
$$

where $O(\delta)$ is small in the accepted linearised sense.

\section{A condition for small angular displacement as $r \rightarrow 0$}

From (5) it is seen that the angular displacement $\theta$ of the pendulum is zero at each of the zeros of $J_{1}(x)$, of which there is just one in the range $0<x<X_{n}$, where $X_{n}$ is the smallest positive zero of $J_{2}(x)$. It follows that, as $r$ decreases, the displacement $\theta$ vanishes just once between its last extremum and the end of the motion. After it vanishes for the last time, $\theta$ approaches its terminal value as indicated in (7), provided that it remains small as $r \rightarrow 0$; for the present it will be assumed that this is the case.

It follows from (7) and (9) that

$$
|\theta| \sim \theta_{1}\left|J_{0}\left(X_{1}\right)\right|^{-1}, \quad r \rightarrow 0,
$$

or, in virtue of (11),

$$
|\theta| \sim\left|J_{0}\left(X_{n}\right)\right|^{-1} O(\delta), \quad r \rightarrow 0,
$$

From tables [2] it is found that

$$
X_{n} \approx 5.136, J_{0}\left(X_{n}\right) \approx-0.1322,
$$

so that (14) is

$$
|\theta| \sim 7.56 \times O(\delta),
$$$$
r \rightarrow 0 \text {. }
$$

The terminal value of $\theta$ will therefore not be small unless the earlier condition (12) is made more restrictive. It is easily seen to be necessary that (12) be replaced by the more stringent condition

$$
\theta=\left|J_{0}\left(X_{1}\right)\right| O(\varepsilon),
$$

$$
x=X \text {, }
$$

where $O(\varepsilon)$ is a quantity which is small in the usual sense, and that the terminal value replacing (14) will then be

$$
|\theta| \sim O(\varepsilon), \quad r \rightarrow 0 .
$$

If (17) is taken as an initial condition for a lengthening pendulum $(V>0)$, the pendulum behaviour will be physically the reverse of that described above for $V<0$, since the angular displacement remains of small magnitude for a lengthening pendulum. The 'reversibility' argument used in the previous section now shows that (16) implies (17) for a shortening pendulum. 
The condition (16) (together with (4)) is therefore a sufficient condition for small angular displacements of a shortening pendulum to continue as $r$ decreases through the whole range $0 \leqq r \leqq l$.

\section{References}

[1] M. N. Brearley, 'The simple pendulum with uniformly changing string length', Proc. Edin. Math. Soc. 15 (Series II) (1966).

[2] E. Jahnke and F. Emde, Tables of functions (New York, 1945).

R.A.A.F. Academy

Victoria 